\title{
Museos y espacios arquitectónicos históricos: la narrativa transmedia como herramienta para la difusión de la información
}

\section{Museums and Historical Architectural Sites: Transmedia Storytelling as a Tool for the Dissemination of Information}

\section{Jennifer Carolina Cruz Barbosa}

Universidad Politécnica de Madrid, jcarolinacbarbosa@gmail.com

\begin{abstract}
Resumen
En la actualidad las nuevas generaciones se encuentran en constante consumo de información mediante diferentes dispositivos electrónicos. Celulares, computadoras, iPads, entre otros se hacen presentes en la vida cotidiana de las personas. Es por esto, que los consumidores esperan recibir información a través de distintos medios, los cuales se complementan y convergen entre sí.

La narrativa transmedia se define como un concepto/ historia que se va complementando e integrando a través de diferentes plataformas y dispositivos. Esto genera que el usuario juegue un papel activo dentro del consumo de información, logrando integrar esta fácilmente a sus conocimientos, teniendo un mejor proceso cognitivo y disfrutando la experiencia. La transmedia se puede erigir desde el estudio de la semiótica, aterrizando el modelo de comunicación para el contexto museístico.
\end{abstract}

Los museos y espacios arquitectónicos históricos se encuentran ante el desafio de adaptar sus exposiciones y documentación al formato digital para seguir compartiendo la cultura a pesar de las limitaciones derivadas del COVID-19. Otra problemática dentro de la gestión del patrimonio cultural es la falta de potencialización de la comunicación de la relevancia de los bienes inmuebles. Estas situaciones, pueden presentarse como una oportunidad para llevar a cabo la adaptación al formato digital con dos objetivos:

1)Continuar siendo centros de información cultural a pesar de la contingencia sanitaria.

2)Proponer formas de comunicación accesibles y atractivas para captar la atención del público, generando un significado memorable y positivo en el contenido que se desea exponer, por medio de la narrativa transmedia.

Para lograr esta implementación, se deben sumar los objetivos que cada museo o espacio arquitectónico tenga individualmente, los cuales pueden ser 
el compartir sus exposiciones permanentes o nuevas colecciones, crear mayor presencia en plataformas digitales, atraer a nuevo público, entre otros. Una vez planteados los objetivos, se prosigue al plan para la ejecución de la transmedia, donde se debe definir la narrativa a establecer, la experiencia deseada en el usuario, la audiencia final, la delimitación de las plataformas, el modelo de negocio y la ejecución.

La transmedia abre la puerta a compartir información relacionada entre sí en múltiples canales digitales. Esto aplicado a los museos, se presenta como una herramienta atractiva, ya que permite mostrar las obras en diferentes plataformas interactivas, mientras que éstas se integran dentro de una narrativa, creando y manteniendo el interés de la audiencia en las exhibiciones, tanto si se encuentran fisicamente en los museos o recintos o no.

Palabras clave: Interacción, HCI, Multimedia, Museología, Cultura.

\begin{abstract}
New generations are in constant consumption of information through different electronic devices. Cell phones, computers, iPads, among others are present in people's daily lives. For this reason, the consumers expect to receive information through different channels, which complement and converge with each other.
\end{abstract}

Transmedia storytelling is defined as a concept/story that is complemented and integrated through different platforms and devices. As a result, the user takes an active role in the consumption of information, easily integrating it to his or her knowledge, having a better cognitive process and enjoying the experience. Transmedia can be built from the study of semiotics, providing the communication model for the museum context.

Museums and historical architectural spaces are facing the challenge of adapting their exhibitions and documentation to the digital format to continue sharing culture, despite the limitations derived from the COVID. Another problem within the management of cultural patrimony is the lack of empowerment of the communication about the relevance of the cultural items. These situations can be presented as an opportunity to implement the adaptation with two objectives:

1)To continue being cultural information centers regardless of the health contingency.

2)To propose accessible and attractive forms of communication to capture the attention of the public and generate a memorable and positive meaning in the content to be exhibited through transmedia narrative. 
To achieve this implementation, the objectives that each museum or architectural space has individually have to be detected, which can be to share their permanent exhibitions or new collections, to increase their presence on digital platforms, to reach new audiences, and others. Once the objectives have been set, the plan for the execution of the transmedia starts, to define which narrative will be established, the desired user experience, the final audience, the delimitation of the platforms, the business model and the execution.

Transmedia opens the door to sharing related information on multiple digital channels. This, applied to museums, is presented as an attractive tool, due to it allows the artworks to be shown on different interactive platforms, while being integrated into a narrative, creating and maintaining the audience's interest in the exhibitions, whether they are physically in the museums or not.

Keywords: Interaction, HCI, Multimedia, Museology, Culture 


\section{Introducción}

El mundo ha experimentado un fenómeno que vino a resaltar la importancia de la tecnología, la comunicación y la necesidad de modernización de diferentes áreas. A causa del esparcimiento del COVID-19, el aislamiento obligatorio y las limitaciones establecidas, la sociedad ha acelerado su adaptación a la era digital para resolver sus necesidades. Diferentes plataformas para video-llamadas, aplicaciones móviles de participación colectiva y recorridos virtuales ahora son parte de la vida diaria.

Esta situación no ha resultado únicamente en proceso de adaptación, también se ha desarrollado un proceso de innovación. Este concepto refleja el trabajo que muchas empresas, personas y grupos sociales han tenido que desarrollar en el último año para poder mantenerse en contacto con sus consumidores y público, continuar ofreciendo sus servicios y productos $\mathrm{y}$ creando un posicionamiento digital.

Es así como a través de la innovación y la transformación, la sociedad ha creado de una situación poco favorable, una oportunidad. Ésta oportunidad también ha sido detectada y trabajada por el sector cultural, especialmente en la rama museística y de recintos arquitectónicos, ya que, al no poder recibir visitas físicas tanto de público nacional como internacional, han tenido que desarrollar nuevas maneras de seguir en contacto con sus visitantes, de conservar su interés y de mantener la información actualizada.

Cabe mencionar que la adaptación y la innovación que se han desenvuelto en el periodo de la pandemia han aprovechado los sistemas de comunicación que son parte de la vida cotidiana de las personas, tales como los teléfonos celulares, las tabletas y las computadoras, por mencionar algunos.

Es aquí donde los investigadores y diseñadores de la comunicación y difusión cultural tienen la oportunidad de ir un paso más allá de lo ya logrado y preguntarse: ¿qué sucede si se utilizan estas distintas plataformas de comunicación para contar la historia de las exposiciones y el arte de una manera interactiva? ¿cómo lograr que los interesados vivan una experiencia inmersiva tanto si se encuentran en los recintos como en sus casas? ¿cómo seguir siendo centros de difusión de información? la respuesta la podemos encontrar en la transmedia storytelling.

La narrativa transmedia (transmedia storytelling) es un concepto relativamente reciente. Si se busca una definición, se puede tomar la propuesta por Henry Jenkins (2007), catedrático de la Universidad de Carolina del Sur y precursor en cultura participativa digital y narrativa, el cual define el concepto como "Un proceso en el que los elementos integrales de una ficción se dispersan sistemáticamente en múltiples canales con el fin de crear una experiencia unificada y coordinada". Es así como se habla de la creación de diferentes contenidos multimedia tales como videos, imágenes, infografías, playlist o pantallas interactivas, que se basan en un tema en concreto, el cual se va uniendo a través de una narrativa. Esto provoca que la propuesta sea atractiva para el público, ya que al querer conocer más sobre la temática y al saber que existe más información desplegada en diferentes plataformas, se va integrando 
cada vez más a la experiencia, hasta llegar al punto en el que se vuelve no solamente consumidor, sino creador de contenido. Así no solamente se logra la aceptación, sino que también se logra la mejor comprensión de los conceptos por parte de los usuarios, en paralelo con un posicionamiento positivo del museo/recinto, gracias a la propuesta de valor.

Junto con Jenkins, se encuentran otros autores quienes han dedicado su trabajo e investigación a las implicaciones de la transmedia. Entre ellos se encuentran Carlos Scolari, profesor investigador de la Universidad Pompeu Fabra en Barcelona, España; Kevin Moloney, profesor en Ball State University's Center for Emerging Media Design and Development, en Estados Unidos, entre otros. Estos autores han aportado información valiosa sobre cómo lograr mejores narrativas con ayuda de las tecnologías y avances digitales que se aparecen día a día; del mismo modo, cada vez más interesados en el tema aportan información sobre cómo mejorar el proceso cognitivo que nace en los espectadores.

Es posible que gran porcentaje de las personas con acceso a la tecnología en algún momento hayan interactuado con contenido propio de la narrativa transmedia. Se tienen por ejemplo a grandes sagas de la literatura como El Señor de los Anillos, Harry Potter y Juego de Tronos, las cuales han sabido extender su universo ficticio a sus fans a través de múltiples plataformas como videojuegos, televisión, cine, exhibiciones y elementos coleccionables, por mencionar solo algunas. Es de esta manera como los espectadores se sienten parte del fenómeno, lo que provoca una mejor relación con la historia y una experiencia memorable. En estos ejemplos se puede notar lo ya mencionado sobre que los espectadores terminan siendo creadores de contenido, dado que dentro de la web existen diferentes wikis (páginas que pueden ser editadas por diferentes personas), dedicadas a teorías, información de personajes y complementos de las historias, gracias al fanatismo alcanzado. Lograr este punto en un ámbito cultural resultaría benéfico para la promoción de obras y que los espectadores comprendan las influencias entre diferentes artistas.

Cabe mencionar que en el área de la comunicación existe otro concepto conocido como crossmedia, el cual, en ocasiones llega a ser confundido con la transmedia. Para lograr la diferenciación se establece que la transmedia crea contenidos pertenecientes a una misma temática y estos se difunden en diferentes plataformas, y si una de estas plataformas no es consumida por el usuario, la historia y el concepto no pierden sentido. Por otro lado, en la crossmedia si una plataforma no es consultada por el usuario, se pierde la secuencia y significado de toda la historia, por lo que la experiencia se queda incompleta, siendo una propuesta en la que todos sus elementos se vuelven dependientes. En la crossmedia, la necesidad de que cada uno de los contenidos tenga que ser explorados puede resultar perjudicial para el usuario ya que se les limita la libertad de consulta, de descubrimiento, de sorpresa y de navegación entre los contenidos. Esto, en contraste, se logra adecuadamente con la transmedia.

La narrativa transmedia existe gracias a la unión de dos posturas: la comunicación - en este caso cultural- y las propuestas tecnológicas. 
Desde la comunicación cultural, se puede estipular que la transmedia se erige desde el estudio de la semiótica, en el que, en este caso, se aterriza el modelo de comunicación en el contexto museístico. De acuerdo con Humberto Eco (1991), "La semiótica se ocupa de cualquier cosa que pueda considerarse como signo. Signo es cualquier cosa que pueda considerarse como sustituto significante de cualquier otra cosa". En otras palabras, las obras de arte o elementos arquitectónicos llegan a formar parte de un grupo de representaciones que dan significado a un lugar, en este caso un museo o un recinto arquitectónico. Es justamente por lo cual se debe impulsar a través de la comunicación transmediática, ya que estos signos -y símboloscuentan la historia de la sociedad y del presente, ya que ilustran el estilo de vida, las tradiciones, la organización social, las creencias y los elementos característicos de un colectivo. Si el público reconoce el mensaje semiótico, comprenderá la influencia que se ha dado entre culturas, los principios de la sociedad que ahora somos y la relación entre signos y objetos.

Uno de los aspectos de la semiótica es la semántica, que se refiere a los sistemas de signos como medio de expresión de un sentido. Es así que, con la semántica, se puede estudiar cómo los objetos presentes en los museos están establecidos de cierta manera con el objetivo de comunicar ideas, pensamientos, sentimientos o conceptos.

Por otro lado, en cuanto a las propuestas tecnológicas, la transmedia va relacionada con la Interacción Humano-Computadora (Human-Computer Interaction, HCI, por sus siglas en inglés), que trasladado al ámbito museístico se puede denominar "diseño de la interacción". Al estudiar la HCI, se descubre que, se han suscitado cuatro etapas que desembocan en la convergencia de plataformas. Durante la década de 1960, se tenía solamente un dispositivo tecnológico para varias personas. Posteriormente en 1980, se logró mayor accesibilidad a estos productos y las personas ya podían disponer de uno en sus hogares. Llegando al año 2000, la sociedad podía disponer de más dispositivos, básicamente un computador y uno de los primeros teléfonos móviles. Finalmente, después del 2010, se habla de diversos dispositivos para una sola persona, entre los cuales podemos encontrar smartphones, tabletas, computadoras, reproductores de música, lectores, relojes inteligentes, entre otros. Es así como se ha dado un giro de $180^{\circ}$ con respecto a la situación de la década de 1960 . Tener esta ventaja en la actualidad debe ser aprovechada, ya que la diversidad de dispositivos significa variedad de maneras de compartir la información y diferentes experiencias que pueden ser explotadas por los museos. Al final, cada uno de los diferentes dispositivos tiene una finalidad primaria que los diferencia de los demás. Hay contenido que resulta mejor consultarlo en un celular que en una computadora, y existen tareas que se desarrollan mejor en un iPad que en un smartwatch. Detectar estas propuestas de valor ayudan a generar un plan de transmedia funcional.

Una vez explicado el término de transmedia y las dos posturas donde se aborda culturalmente, es momento de pasar al estudio de la comunicación de la información. Se manejan dos tipos de contenido, los expuestos por el museo y los generados por los visitantes. 
Respecto al primer tipo de contenido, para definir cómo serán expuestos al público es fundamental conocer a este último. Al momento de aplicar un plan transmedia en un museo o en un recinto arquitectónico, se encuentra una gran variedad de perfiles de visitantes y de interesados. Se presenta, por ejemplo, la familia conformada por padres de mediana edad y niños, también se debe considerar a los adolescentes interesados en la cultura, así como a la gente de la tercera edad que han acudido a museos durante toda su vida. Tener un público tan amplio provoca que se deban desarrollar diferentes herramientas que mantengan la atención de quienes ya conocen la oferta cultural y que despierte la curiosidad de aquellos ajenos a ella. La sociedad cambia constantemente, sus intereses van variando día a día al igual que sus comportamientos. Por ello, los comunicadores y precursores de la cultura deben evolucionar a la par del público para seguir hablando el mismo idioma.

Enfocándose en el segundo tipo de contenidos, que son los generados por los usuarios en el contexto museístico, se debe notar que la mayoría de los visitantes recorren las exhibiciones y lugares con sus dispositivos celulares en la mano, debido a que durante la visita toman fotografías de los elementos que captan su atención, buscan en internet más información de las obras o comparten una story en sus redes sociales. Son justamente estos tipos de interacciones de donde se pueden obtener propuestas de valor para potencializar la información que contienen las obras artísticas. Se trata de utilizar la tecnología a favor de la cultura. Esta misma interacción del público con la obra genera una participación colectiva, ya que las diferentes plataformas digitales existentes provocan un intercambio de comunicación entre personas que, aunque no tengan una relación familiar o de amistad, comparten el mismo interés e inician un diálogo y trueque de ideas al respecto. De esta manera, se logra entre los visitantes e interesados, el principal objetivo de los museos y espacios arquitectónicos históricos, que es comunicar, difundir y divulgar información.

Sin embargo, digitalizar los espacios no es una tarea fácil, exige una adaptación de ejecución, planeación e ideología dentro de los responsables del campo de trabajo. En opinión de Joan Santacana y Carolina Martín (2012), autores de "Manual de museografía interactiva": "son todavía una exigua minoría los museos que se plantean la interactividad como un concepto global, casi como una actitud o una necesidad para las exposiciones y el tratamiento del patrimonio. Ocurre con la interactividad como ocurría antes con los audiovisuales”.

Lograr la interactividad de contenidos y la digitalización significa mayor accesibilidad de la información para el público interesado. Francisca Hernández (2011) en su obra "El museo como espacio de comunicación" comparte lo siguiente al respecto:

"Si pretendemos que los museos digan algo significativo a los visitantes del siglo XXI es necesario que su forma de presentar y de exponer las colecciones sea más abierta, interactiva e inclusiva. Ya no sirven los viejos esquemas decimonónicos, donde los museos exponían sus obras de manera monolítica de cara a un público que nada tenía que decir o aportar porque era un mero espectador pasivo de las mismas". 
Estos puntos referentes a la situación actual de la comunicación y la narrativa en los espacios culturales representan un reto que debe tenerse presente al momento de desarrollar la transmedia storytelling dentro de los museos y patrimonio arquitectónico. Este reto debe ser superado para lograr resultados positivos que comprueben las ventajas de la modernización museística.

\section{Objetivos}

Pero ¿cómo se implementa la transmedia? A través de un plan de acción que vea por la adaptación de los contenidos y del museo propio hacia la era digital con base en dos objetivos:

1) Que los museos y espacios arquitectónicos sigan siendo centros de información cultural a pesar de la contingencia sanitaria.

2) Proponer formas de comunicación accesibles y atractivas para captar la atención del público, generando un significado memorable y positivo en el contenido que se desea exponer, por medio de la narrativa transmedia.

\section{Desarrollo de la innovación}

Teniendo como base los objetivos, el plan de acción se puede dividir en seis pasos para lograr el éxito de la propuesta.

En primer lugar, se tiene que definir la audiencia final. En el contexto de los museos, el perfil de los consumidores es muy amplio, ya que involucra a las personas conocedoras del tema y a las que son completamente ajenas, a interesados y a acompañantes. Eso sin contar la diferencia de edades, de nivel socioeconómico y de nacionalidad. En esta parte, se puede proponer que cada una de las plataformas esté enfocada indirectamente a un posible arquetipo de espectador. De esta manera, se alcanza una cobertura global de los intereses. Por ejemplo, a los adolescentes les puede interesar el contenido disponible en una aplicación, a los adultos jóvenes les cautiva lo expuesto en el sitio web, mientras que las personas de tercera edad prefieren los datos impresos.

En esta fase se puede implementar el Diseño Centrado en el Usuario, con el cuál a través del análisis del contexto, la definición de requisitos, el diseño y la evaluación de la propuesta, siguiendo en todo momento un proceso iterativo, se puede lograr la satisfacción y la experiencia positiva de los usuarios, la cual se reflejará en buenos resultados cuantitativos y cualitativos.

En segundo lugar, se desarrolla la narrativa que se empleará dentro de la transmedia. Esta narrativa, no debe ser ajena al espectador, debe seguir un modelo donde éste pueda recordar sus propias experiencias para lograr así una conexión emocional con la obra. Al lograr esta conexión, se está potencializando que la experiencia tenga un significado memorable, lo que es pieza clave para facilitar el proceso cognitivo. Cuando una persona solamente aprecia los elementos culturales sin crear significados, se queda en un estado pasivo y la relación es 
carente. Se debe buscar que la persona recuerde, identifique, relacione y comparta su nuevo conocimiento, que se convierta en un consumidor potencializado.

El uso de micro relatos relacionados con el arte es una herramienta que se debe explorar a fondo. Estos se pueden encontrar de manera ordenada o de consulta flexible en las diferentes plataformas, disponibles para los usuarios. Dichos micro relatos brindan las herramientas cognitivas necesarias que ayudan a tener mayor comprensión y claridad de la cultura. Retomando que las obras de arte y recintos arquitectónicos tratan de comunicar ideas, sentimientos o situaciones, se debe utilizar ello para la creación de la narrativa.

En una tercera etapa, se debe desarrollar y plantear la experiencia deseada en los usuarios. Actualmente, las exposiciones e información museística han sido cuestionadas por los visitantes, debido a que ellos esperan que los canales de comunicación les permitan tener un rol activo y participativo, incluso de intérprete. Si esta necesidad se le llega a cumplir a los usuarios, no solamente se les demuestra que sus opiniones han sido escuchadas, sino que, además, la relación con las obras o datos se vuelven significativa, cercana y envolvente.

Asimismo, es importante que en esta experiencia los usuarios se sientan envueltos en la ambientación del lugar expositivo o patrimonial a pesar de no poder encontrarse físicamente en él, por lo que se debe procurar la fidelización no solo a los objetos exhibidos, si no al espacio arquitectónico donde se encuentran, para de esta manera no solamente compartir el contenido, sino también el contexto. Esto ayuda a procurar la correcta percepción y reconocimiento de los objetos.

En cuanto a la experiencia buscada cuando las personas sí se encuentran dentro del recinto cultural, se debe lograr que las interfaces de la propuesta transmedia contengan estímulos sensoriales latentes, de manera que los visitantes detecten los elementos interactivos, los comprendan intuitivamente y se sientan con la libertad de interrelacionarse con ellos. La interacción debe ser consistente y correctamente enfocada en la exposición.

Después de establecer cuál es la experiencia que se desea alcanzar, se da inicio a la cuarta parte del desarrollo del proyecto, que refiere a la delimitación de las plataformas a utilizar. Se debe iniciar planteando qué elementos se van a digitalizar, qué interfaces se mantendrán en un nivel tradicional y cómo se van a establecer affordances perceptibles que inviten a las personas a interactuar con las exposiciones y a ser parte de una participación colectiva. Existen diferentes opciones multimedia para desplegar la información. En el caso de las interfaces presentes de manera física en los museos o recintos arquitectónicos, se pueden emplear los beacons (dispositivos basados en tecnología bluetooth), pantallas de tacto, códigos QR e interfaces tangibles (objetos físicos con sensores), por mencionar algunos. Por otro lado, entre las plataformas que se pueden proponer para expandir la información fuera del museo/recinto, la principal herramienta, actualmente, son las redes sociales. Sin embargo, el contenido no debe limitarse solamente a Instagram, Facebook y Twitter. Se pueden realizar intervenciones gráficas en espacios públicos, productos editoriales coleccionables, figuras relacionadas con las obras o aplicaciones móviles. Existen más de veinte interfaces y touchpoints que pueden ser utilizados por la narrativa transmedia. Por lo tanto, el equipo que 
lleve a cabo el proyecto de comunicación cultural tiene la responsabilidad de saber identificar cuáles son las adecuadas para llegar al público deseado, que sean posibles de desarrollar tomando en cuenta las capacidades y limitantes del museo y que van a arrojar un resultado llamativo, usable y accesible.

El quinto punto por cumplir es delimitar el modelo de negocio a implementar. La parte del presupuesto fijará qué tipo de interfaces se pueden construir, ya que los costos de producción son dependientes del nivel de tecnología deseado. La inversión en el ámbito cultural apoya a la colocación de museos y recintos arquitectónicos en el interés de la sociedad y, si se desarrolla correctamente, capta la atención de público extranjero. Los beneficios de destinar fondos a la promoción de la cultura se verán reflejados en resultados positivos tanto cuantitativos (mayor número de visitas, incremento de seguidores) como cualitativos (popularidad, recomendación, posicionamiento).

Finalmente, todas estas fases cobran vida en la ejecución. Es en esta última etapa donde la intersección de la tecnología con la cultura y la sociedad llevará a los museos y recintos a poder comunicarse con su público logrando un enfoque en la información y el compromiso con los objetos exhibidos.

\section{Resultados}

Al momento de llevar el plan a la ejecución, los puntos claves que se deben considerar son los medios, los usuarios y el proceso de interacción existente entre estos. Tener estos tres elementos en conciencia ayudarán a garantizar el correcto desarrollo de la transmedia, logrando la atención del consumidor y un proceso cognitivo perdurable.

Es necesario resaltar que los beneficios de la aplicación de la narrativa transmedia son medibles y concebibles. Para realmente alcanzarlos, se requiere de un equipo de trabajo que sepa delimitar correctamente las plataformas y los objetivos que perseguirá cada una. El empleo de la tecnología no garantiza la satisfacción de las necesidades y deseos de un usuario, por lo que la inversión debe ser correctamente empleada en canales de comunicación que sumen, sean accesibles y al alcance del público. No servirá el implementar una interfaz de realidad aumentada dentro de un museo si solamente una mínima parte de los usuarios podrá hacer uso de ella, o si se implementa únicamente por considerarla una herramienta novedosa. Los canales de comunicación seleccionados deben llevar a los usuarios siempre al razonamiento y a la reflexión con base en la interacción vivida.

\section{Conclusiones}

En conclusión, al ser parte de espacios destinados a la comunicación y difusión de la información, tal como lo son los museos y espacios arquitectónicos, se debe estar en constante actualización sobre los canales de comunicación existentes para mantener el contacto e interés de los visitantes frecuentes y atraer la atención de los potenciales. Los museos suelen ser considerados lugares tradicionales donde las personas solamente son 
espectadores y no toman un papel activo. Seguir viendo la cultura de esta manera, ajena a una sociedad vibrante y dinámica, crea barreras y da el mensaje erróneo. Es momento de innovar, de crear conexiones reales y experiencias culturales. La interacción digital con el arte potencializará su recepción en el espectador, y dará pie a una interacción social centrada en las exposiciones. La narrativa transmedia es una herramienta que impulsa la comunicación, la participación y la creatividad dentro de los espacios museísticos gracias a las herramientas digitales y a las tecnologías emergentes, por lo que debe ser empleada y se debe seguir investigando para detectar nuevas propuestas de valor.

\section{Referencias}

DIAPER, D. (1989). Rask Analysis for Human-Computer Interaction. Inglaterra: Ellis Horwood. ECO, U. (1991). Tratado de semiótica general. Barcelona: Lumen.

FERNÁNDEZ, L. (1999) Museología y museografía. Barcelona: Ediciones Del Serbal.

HERNÁNDEZ, F., 2011. El Museo Como Espacio De Comunicación. 2nd ed. Austrias: Ediciones Trea, S. L.

JENKINS, H. (2007). Transmedia Storytelling 101.

$<$ http://henryjenkins.org/blog/2007/03/transmedia_storytelling_101.html > [Consulta: 5 de febrero de 2021]

JENKINS, H (2008) Convergence Culture. Barcelona: Grupo Planeta. KOLKO, J. (2010). Thoughts on Interaction Design. Burlington: Elsevier Inc.

PRATTEN. R. (2011) Getting Started in Transmedia Storytelling - A Practical Guide for Beginners. London: CreateSpace Independent Publishing Platform.

MULLET, K., \& SANO, D. (1995). Designing Visual Interfaces. California: SunSoft Press.

Los museos interactivos como mediadores pedagógicos (2005) vol. 26, Sinéctic.

SANTACANA, J., \& MARTÍN PIÑOL, C. (2012). Manual de museografía interactiva. Austrias: Ediciones Trea, S. L.

SEARS, A., \& JACKO, J. (2009) Human-Computer Interaction, fundamentals. Boca Ratón: Taylor y Francis Group.

SCOLARI, C. (2008) Hipermediaciones. Barcelona: Editorial GEDISA. SCOLARI, C. (2013). Narrativas Transmedia (1st ed.). Barcelona: Grupo Planeta. 